OPEN ACCESS

Edited by:

Fei Yan,

Shenzhen Institutes of Advanced

Technology (CAS), China

Reviewed by:

Yihan Chen,

Huazhong University of Science and

Technology, China

Feng Wang,

Xinxiang Medical University, China

*Correspondence:

Qiao Hu

xyyxyhq@163.com

Zhenzhou Li

lizhenzhou2004@126.com

tThese authors have contributed equally to this work.

Specialty section:

This article was submitted to Translational Pharmacology,

a section of the journal

Frontiers in Pharmacology

Received: 30 January 2019

Accepted: 20 May 2019

Published: 19 June 2019

Citation:

Zhou Y, Song Z, Hu Q, Ji X, Zheng H, Wang $X$ and Li Z (2019) Evaluation of the Expression of Matrix Metalloproteninases-1 of Laryngeal Squamous Cell Carcinoma by Ultrasound Molecular Imaging

Front. Pharmacol. 10:655

doi: 10.3389/fphar.2019.00655

\section{Evaluation of the Expression of Matrix Metalloproteinase-1 of Laryngeal Squamous Cell Carcinoma by Ultrasound Molecular Imaging}

\author{
Yi Zhou ${ }^{1,5 t}$, Zhuqing Song ${ }^{2 \dagger}$, Qiao $\mathrm{Hu}^{3 *}$, Xiaojuan $\mathrm{Ji}^{4}$, Hongyu Zheng ${ }^{3}$, Xiaoyan Wang ${ }^{3}$ \\ and Zhenzhou $\mathrm{Li}^{5 *}$ \\ ${ }^{1}$ GuangZhou University School of Medicine, GuangZhou, China, ${ }^{2}$ Department of Breast Surgery, Peking University Shenzhen \\ Hospital, Shenzhen, China, ${ }^{3}$ Department of Ultrasound, The People's Hospital of Guangxi Zhuang Autonomous Region, \\ Nanning, China, ${ }^{4}$ Department of Cardiology, Children's Hospital of Chongqing Medical University, Chongqing, China, \\ ${ }^{5}$ Department of Ultrasound, The Second People's Hospital of Shenzhen, The First Affiliated Hospital of Shenzhen University, \\ Shenzhen, China
}

Purpose: The aims of this study were to evaluate the expression of matrix metalloproteinase-1 (MMP-1) on laryngeal squamous cell carcinoma (LSCC) and improve the early diagnosis rate via ultrasound molecular imaging (USMI).

Methods: The microsized MMP-1-targeted microbubbles $\left(\mathrm{MB}_{\mathrm{MMP}-1}\right)$ and the control MBs $\left(\mathrm{MB}_{\mathrm{IgG}}\right)$ based on perfluorocarbon-filled lipid-shelled MBs were constructed and characterized. The in vitro binding experiment was performed with human epidermoid laryngeal cancer cells (HEp-2) and tested the binding efficiency of $\mathrm{MB}_{\mathrm{MMP}-1}$ and $\mathrm{MB}_{\mathrm{IgG}}$. In the in vivo study, the LSCC model was established in 10 mice. The $\mathrm{MB}_{\mathrm{MMP}-1}$ and $\mathrm{MB}_{\mathrm{IgG}}$ were randomly injected into tumor-bearing mice via the tail vein at Day 7 , Day 12, and Day 17 to dynamically evaluate the differential targeted enhancement (dTE) signals via USMI. Subsequent immunofluorescence analysis was used for confirmation of MMP-1 expression.

Result: The effective adhesion rate of $\mathrm{MB}_{\mathrm{MMP}-1}$ and $\mathrm{MB}_{\mathrm{IgG}}$ to HEp-2 was $298.42 \pm 16.57$ versus $12.38 \pm 3.26$ bubbles/per field in vitro experiment, which shows a significant difference $(P<0.01)$. The in vivo ultrasound molecular imaging $(\mathrm{USMI})$ results demonstrated that dTE signal intensity from $\mathrm{MB}_{\mathrm{MMP}-1}$ was significantly higher than that from the $\mathrm{MB}_{\mathrm{IgG}}$ at Day 7, Day 12, and Day 17 (Day 7, $41.21 \pm 15.00$ versus $2.25 \pm 0.6$ a.u., $P<0.05$; Day $12,124.64 \pm 5.19$ versus $11.13 \pm 1.13$ a.u., $P<0.05$; Day $17,332.01 \pm 64.88$ versus $42.99 \pm 11.9$ a.u., $P<0.01)$. Moreover, immunofluorescence analysis further confirmed the expression of MMP-1 in LSCC with a gradual increase with the tumor growth.

Conclusion: $\mathrm{MB}_{\mathrm{MMP}-1}$ could be a potential probe that can be used in the early diagnosis of LSCC by USMI.

Keywords: ultrasound molecular imaging, matrix metalloproteinase-1, laryngeal squamous cell carcinoma, targeted microbubbles, vasculogenic mimicry 


\section{INTRODUCTION}

Laryngeal squamous cell carcinoma (LSCC) is one of the common malignant tumors of the head and neck. Recent data from global epidemiology of head and neck cancers show that more than 245,000 new cases of laryngeal cancer will be expected by 2030 (Gupta et al., 2016). Although great significant progress has made in the diagnosis, its early diagnosis rate of LSCC still remains to be raised. Microbubble-based ultrasound molecular imaging (USMI) shows significant potential in the early diagnosis of tumors. Ultrasound contrast agents (UCAs) such as microbubbles (MBs) can emit significantly stronger acoustic signals under an appropriate sonic energy excitation, making them several thousand times more reflective than normal body tissues. Through designing ultrasonic contrast agents with specific molecular markers, USMI probes can be obtained and used to visualize molecular and genetic alterations of diseased cells, and to monitor the genesis and development of certain diseases. Previously, researchers have demonstrated that the MBs combined with antibodies or peptides, which can bind to vascular endothelial growth factor (VEGR) or av $\beta 3$ integrin, and enhanced the contrast ultrasonography quality in LSCC via USMI (Paolo et al., 2006; Hu et al., 2016). However, in malignant LSCC tumors, the expression of EGFR or av $\beta 3$ integrin was commonly not at a high level (Kumar, 2003; Gino et al., 2010).

Matrix metalloproteinase-1 (MMP-1), a member of the family of MMPs, plays crucial roles in vascular formation and remodeling via degrading vascular membrana basilaris and extracellular matrix (ECM) proteins (Raffetto and Khalil, 2008). In addition, several studies have confirmed that the MMP-1 is specially associated with LSCC growth, local invasion, and metastasis (Tsukifuji et al., 1999). Upile et al., (2011) had claimed that the angiogenesis of LSCC was not only a unique means to nourish tumor tissues. Specially, it generated "micro-vascular" channels without the composition of endothelial cells at the early stage. This event was known as vascular mimicry (VM) in which endotheliallike cells are transdifferentiated by their own stem cells. More importantly, both angiogenesis and VM as a part of tumor microenvironment are coordinately providing tumor initiation and progression, and the latter one even seriously contributed to the invasion of LSCC (Upile et al., 2011; Balkwill et al., 2012). In view of above-mentioned reasons, we believed that the biomarker of MMP-1 would provide a potential evaluation of its progress in LSCC biology, thus allowing for the design of new diagnostics and therapeutics for early cancer diagnosis and treatment.

To date, the positive rate of conventional medical imaging examination, such as computed tomography (CT) and magnetic resonance imaging (MRI), in detecting LSCC is still low in detection of LSCC (Castelijns and Mw, 1993; Yamazaki et al., 2008; Norling et al., 2014; Righi et al., 2015). USMI is a multifunctional and famous medical imaging tool for the detection and visualization of cancer-related biomarkers in early disease diagnosis (Pysz et al., 2010; Yan et al., 2011; Feng et al., 2013; Wood and Sehgal, 2015). In the past decades, MBs with different types of shells composed of phospholipids or polymers and gas cores (perfluorocarbon, nitrogen, sulfur hexafluoride, or air) had been applied in USMI and performed good contrast enhancement (Kiessling et al., 2009; Abou-Elkacem et al., 2016). Some special biomarkers of ligands such as integrin and Vascular cell adhesion molecule-1 (VCAM-1) or EGFR can be tightly bound on the surface of MBs for a better specificity diagnosis (Fabian et al., 2012). Recently, it has been successfully applied to various diseases such as vascular plaque and tissue inflammation, but its potential use in the diagnosis of LSCC has not been reported. In our study, we preliminarily developed the $\mathrm{MB}_{\mathrm{MMP}-1}$ and dynamically evaluated the expression of MMP- 1 separately at Day 7, Day 12, and Day 17 of LSCC progression in vivo by USMI.

\section{MATERIALS AND METHODS}

\section{Preparation of $\mathrm{MB}_{\mathrm{MMP}-1}$}

$\mathrm{MB}_{\mathrm{MMP}-1}$ and control $\mathrm{MB}_{\mathrm{IgG}}$ were prepared according to a previous report (Stieger et al., 2010). In brief, DSPC: DSPEPEG2000:DSPE-PEG2000-biotin (Avanti Polar Lipids, Alabaster, AL, USA) (molar ratios = 9:0.5:0.5) was blended in chloroform, and the solvent was removed under nitrogen flow at room temperature, followed by vacuum treatment over $2 \mathrm{~h}$. The dried blends were hydrated at $60^{\circ} \mathrm{C}$ with phosphate-buffered saline (PBS) and sub-packaged into vials ( $1 \mathrm{~mL}$ each vial). After that, perfluoropropane $\left(\mathrm{C}_{3} \mathrm{~F}_{8}\right.$; Flura, Newport, TN, USA) was added, and the admixture was mechanically vibrated for $45 \mathrm{~s}$. After that, $\mathrm{MB}_{\mathrm{MMP}-1}$ contrast group was prepared by incubating these biotinylated MBs with excess avidin, followed by adding a given proportion $\left(50 \mu \mathrm{g} \times 10^{8} \mathrm{MBs}\right)$ of biotinylated anti-mouse MMP- 1 monoclonal antibody (Novus Biologicals, Colorado, USA). Incubation at room temperature for $15-30 \mathrm{~min}$ and washing three to four times by centrifugation $(400 g)$ were necessary for avidin or antibody linkage. The biotinylated IgG antibody (Novus Biologicals, Colorado, USA) was used instead of MMP-1 to connect with biotinylated MBs in the same way to obtain $\mathrm{MB}_{\mathrm{IgG}}$ in the control group.

\section{Characterization of $\mathrm{MB}_{\mathrm{MMP}-1}$}

The surface morphology of $\mathrm{MB}_{\mathrm{MMP}-1}$ was investigated using a microscope (Leica DMI3000 B). Fluorescent microscopic examination was performed to verify the anti-mouse MMP-1 monoclonal primary antibody conjugation efficiency of $\mathrm{MB}_{\mathrm{MMP}-1}$ according to fluorescent intensity of fluorescein isothiocyanate (FITC)-conjugated goat anti-mouse second antibody (St. Louis, MO, USA) under a fluorescent microscope (Olympus, Tokyo, Japan). $\mathrm{MB}_{\mathrm{MMP}-1}$ average size, distribution, and differential intensity were evaluated three times for each sample using a diameter limit of $0.5 \mu \mathrm{m}$ (AccuSizer 780; Particle Sizing Systems, Santa Barbara, CA, USA).

\section{Cell Culture}

Human epidermoid laryngeal cancer cells (Procell Life, Wuhan, China) (HEp-2) were cultured in Dulbecco's modified Eagle medium (DMEM) supplemented with $10 \%$ fetal bovine serum (FBS), 1\% L-glutamine, and 1\% penicillin-streptomycin. The cell cultures were maintained in a humidified atmosphere of $5 \% \mathrm{CO}_{2}$ at $37^{\circ} \mathrm{C}$ with the medium changed every other day. 


\section{Tumor Model}

All animal studies were approved by the Institute's Animal Care and Use Committee of Shenzhen Second People's Hospital, Guangzhou University School of Medicine, China. The methods were carried out in accordance with the approved guidelines. Nude male Balb/c mice (6- to 8-week-old, body weight $20 \pm 1.5 \mathrm{~g}$ ) were supplied by Beijing Vital River Laboratory Animal Technology Co. Ltd. All mice were housed on a 12:12 light:dark cycle with free access to food and water. HEp- 2 cells $\left(1 \times 10^{6} /\right.$ each mouse $)$ dissolved in $100 \mu \mathrm{L}$ of PBS were injected into the left oxter of nude male Balb/c mice. Tumor-bearing mice were used for USMI when they had developed at Day 7, Day 12, and Day 17 after inoculation.

\section{In Vitro Imaging Performance of $\mathrm{MB}_{\mathrm{MMP}-1}$}

$\mathrm{MB}_{\mathrm{MMP}-1}$ with concentrations of $1 \times 10^{5}, 1 \times 10^{6}$, and $1 \times 10^{7} \mathrm{MBs} / \mathrm{mL}$ was placed in agar gel holes. The US imaging performance of MBs was detected from the side of the agar gel with VisualSonics Vevo2100 (VisualSonics, Inc., Toronto, Canada) to determine the intensity of the ultrasound signal of the MBs with concentrations of $1 \times 10^{5}, 1 \times 10^{6}$, and $1 \times 10^{7} \mathrm{MBs} / \mathrm{mL}$.

\section{Binding Specificity of $\mathrm{MB}_{\mathrm{MMP}-1}$ to Human Epidermoid Laryngeal Cancer Cells}

In a 6 -well plate $\left(1 \times 10^{5}\right.$ cells per well $), 1 \times 10^{5} \mathrm{HEp}-2$ cells were cultured overnight. The next day, the culture medium was discarded, and the cells were washed three times with PBS, and then $1 \times 10^{8} \mathrm{MBs} / \mathrm{mL}$ of $\mathrm{MB}_{\mathrm{MMP}-1}$ or $\mathrm{MB}_{\mathrm{IgG}}$ was added to the cells and incubated to the plate for 5-6 min. After being washed three to five times with $\mathrm{PBS}$, the binding efficiency of $\mathrm{MB}_{\mathrm{MMP}-1}$ and control $\mathrm{MB}_{\mathrm{IgG}}$ to cells was examined under an inverted microscope (Olympus, Tokyo, Japan). At the same time, the competitive binding inhibition experimental group (preblocking MMP-1 receptors by adding an excess of free MMP-1 monoclonal antibody) was also set up to study the binding specificity of $\mathrm{MB}_{\mathrm{MMP}-1}$ to $\mathrm{HEp}-2$.

\section{In Vivo Ultrasound Molecular Imaging}

USMI was conducted as described previously (Stieger et al., 2010). Briefly, the mice were kept anesthetized by continuous inhalation of $2 \%$ isoflurane in oxygen at $2 \mathrm{~L} / \mathrm{min}$ on a heated stage during scanning. US imaging was performed using a dedicated smallanimal high-resolution USMI system (Vevo2100, VisualSonics) equipped with an $18-\mathrm{MHz}$ high-frequency nonlinear transducer. All imaging parameters (grain, $30 \mathrm{~dB}$; focal depth, 2-4 mm; transmit power, $10 \%$; MI, 0.1 ) were kept constant during all imaging sessions. $\mathrm{MB}_{\mathrm{MMP}-1}$ or $\mathrm{MB}_{\mathrm{IgG}}\left(5 \times 10^{8} \mathrm{MBs}\right.$ in $200 \mu \mathrm{L}$ of PBS) was administered via the tail vein of the mice in random order to minimize bias, and injections were separated by at least 30 min to allow clearance of MBs from the blood circulation. To distinguish the acoustic signal from MBs that had adhered to MMP-1 receptors from the signal from freely circulating MBs, a destruction/replenishment approach was used in this study. In brief, after injection of MBs and waiting for $7 \mathrm{~min}$, approximately 200 ultrasonographic frames of the tumor were acquired at a temporal resolution of $12 \mathrm{~s}$. Then a high-power ultrasound destruction sequence was applied for $1 \mathrm{~s}$ to destroy the MBs. After the destruction pulse, another set of movie $(\approx 200$ frames) was acquired. Movie processing and quantification were performed using Vevo2100 built-in software of CQ and relying on two sets of movie: a pre-destruction set and a postdestruction (background) data set. The post-destruction movie signals were subtracted from the pre-destruction signals. The difference in movie signal intensity between pre-destruction and post-destruction ultrasonographic frames was calculated and expressed as movie intensity amplitude.

\section{Imaging Data Analysis}

The imaging data sets of all mice were analyzed by Vevo 2100 built-in software CQ. Regions of interest were drawn covering the entire area of the tumor. The movie signal intensity from attached MBs was assessed by calculating an average for pre-destruction and post-destruction imaging signals and subtracting the average post-destruction signal from the average pre-destruction signal. The subtracted signal, so-called differential targeted enhancement (dTE), was colored red and then displayed as a colored overlay on the contrast-mode images.

\section{Immunofluorescence}

Tumor-bearing mice were euthanized after USMI, and the subcutaneous tumors were excised, embedded in optimal cutting temperature compound, and frozen in dry ice. Frozen blocks were sectioned at $5 \mu \mathrm{m}$ (CM1950, Leica, Heidelberg, Germany) and mounted on glass slides for immunofluorescence staining. A double-staining procedure was employed to visualize MMP-1 expression on tumor cells. The following method was used for mouse MMP-1 staining. First, paraffin sections were successively dewaxed with xylene I for $15 \mathrm{~min}$ and xylene II for $15 \mathrm{~min}$; hydrated with absolute ethanol I for $5 \mathrm{~min}$, absolute ethanol II for $5 \mathrm{~min}, 85 \%$ ethanol for $5 \mathrm{~min}$, and $75 \%$ ethanol for $5 \mathrm{~min}$; and afterwards washed with distilled water. Second, these were subsequently blocked with $5 \%$ goat serum for $30 \mathrm{~min}$ at room temperature. And then the slides were co-incubated with rabbit anti-mouse MMP-1 primary antibody with dilution 1:1,500 ratio (Google Biotechnology Co., Ltd, Wuhan, China) overnight at $4^{\circ} \mathrm{C}$ and visualized by using $\mathrm{Cy} 3$-conjugated goat anti-rabbit second antibody (Servicebio, Wuhan, China) in a 1:300 ratio (Google Biotechnology Co., Ltd, Wuhan, China). Next, the slides were placed in PBS ( $\mathrm{pH} 7.4$ ) on as decolorization shaking table for $5 \mathrm{~min}$. After the drying process, dihydrochloride (DAPI) dye was added to the slides, and samples were protected from light and incubated for $10 \mathrm{~min}$. Finally, fluorescent images were acquired at $\times 400$ magnifications with a laser scanning confocal microscope (TCS SP5, Leica, Germany). And then the correlation analysis between the signal intensity of USMI and mean optical density (/pixel) of MMP-1 expression was performed separately at Day 7, Day 12, and Day 17.

\section{Statistical Analysis}

Quantitative data were expressed as the means and standard deviation (mean $\pm S D$ ). Data from two independent samples were 
analyzed with Student's $t$ test. Analysis of variance (ANOVA) was used to determine the significance of differences in multiple comparisons. A $P$ value of less than 0.05 was considered statistically significant. Statistical analyses were performed using Statistical Product and Service Solutions (SPSS) software version 13.0 (SPSS Inc, Chicago, IL, USA).

\section{RESULTS}

\section{Characterization of $\mathrm{MB}_{\mathrm{MMP}-1}$}

Figure 1 shows a schematic illustration of $\mathrm{MB}_{\mathrm{MMP}-1}$. Microscopic image analysis at high magnification revealed that the resulting $\mathrm{MB}_{\mathrm{MMP}-1}$ has regular spherical morphology, transparent center, and good dispersion with no adherence to each other (Figure 2A). The FITC-conjugated $\mathrm{MB}_{\mathrm{MMP}-1}$ shows bright green fluorescence under fluorescence microscope (Figure 2B), indicating the successful conjugation of anti-MMP-1 antibodies onto the surface of MBs. Mean size distributions of the $\mathrm{MB}_{\mathrm{MMP}-1}$ are shown in Figure $2 \mathrm{C}$ by AccuSizer 780, revealing that the mean diameter of the $\mathrm{MB}_{\mathrm{MMP}-1}$ was centered at $1.11 \pm 0.10 \mu \mathrm{m}$.

\section{In Vitro Imaging Performance of $\mathrm{MB}_{\mathrm{MMP}-1}$}

To confirm the imaging performance of $\mathrm{MB}_{\mathrm{MMP}-1}$ as a contrast agent for USMI, three different concentrations of $\mathrm{MB}_{\mathrm{MMP}-1}$ were assessed in vitro via contrast imaging mode (Figure $\mathbf{3 A}, \mathbf{B}, \mathbf{C}$ ). We found the three samples' echoes are relatively well distributed. In addition, with the increase of the concentration of $\mathrm{MB}_{\mathrm{MMP}-1}$, the signal intensity of contrast imaging increases. In particular, we found that the best performance of contrast imaging was at $1 \times 10^{7} \mathrm{MBs} / \mathrm{mL}$. Then, we quantified the average signal intensity of different concentrations of $\mathrm{MB}_{\mathrm{MMP}-1}$, revealing $4.11 \pm 1.37$, $24.03 \pm 2.06$, and $90.72 \pm 4.92$ a.u. at $1 \times 10^{5}, 1 \times 10^{6}$, and $1 \times 10^{7}$ $\mathrm{MBs} / \mathrm{mL}$, respectively (Figure 3D).

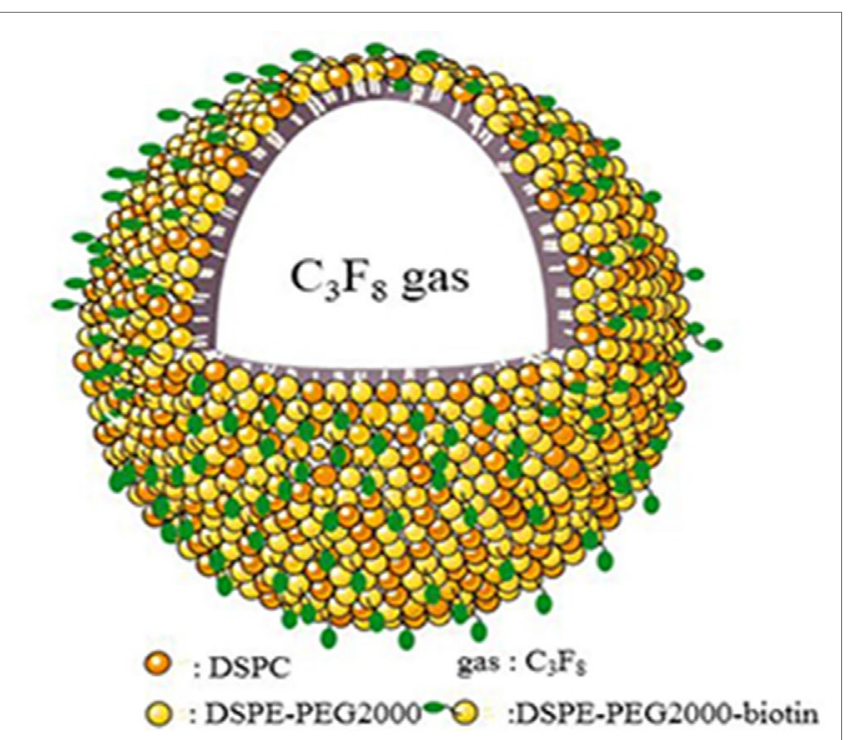

FIGURE 1 | Schematic illustration of $\mathrm{MB}_{\mathrm{MMP}-1}$. DSPC =

distearoylphosphatidylcholine; DSPE = distearoylphosphatidylethanolamine; PEG2000 = polyethylene glycol 2000.

\section{Binding Specificity of $\mathrm{MB}_{\mathrm{MMP-1}}$ to Human Epidermoid Laryngeal Cancer Cells}

To ensure the binding specificity of the $\mathrm{MB}_{\mathrm{MMP}-1}$, we further conducted the binding specificity of $\mathrm{MB}_{\mathrm{MMP}-1}$ or $\mathrm{MB}_{\mathrm{IgG}}$ with the HEp-2 by comparing with the group that was pre-incubated with free anti-MMP-1 antibodies for 5-6 min. In bright field, our results (Figure 4A) showed that the control $\mathrm{MB}_{\mathrm{IgG}}$ only had a small number of non-targeted binding specificity on the HEp-2 surface with $12.38 \pm 3.26 \mathrm{MBs} /$ per field, while the $\mathrm{MB}_{\mathrm{MMP}-1}$ could specifically bind to the HEp-2 with $298.42 \pm 16.57 \mathrm{MBs} /$ per field (Figure 4B) (298.42 \pm 16.57 versus $12.38 \pm 3.26 \mathrm{MBs} /$ per field, $P<0.01)$. And as for the pre-blocking group by an excess of free anti-MMP-1 monoclonal antibody, it was found that the number of $\mathrm{MB}_{\mathrm{MMP}-1}$ attached to HEp-2 was significantly decreased with $37.3 \pm 6.94 \mathrm{MBs} /$ per field (Figure 4C) (298.42 \pm 16.57 versus $37.3 \pm 6.94 \mathrm{MBs} /$ per field, $P<0.01)$. Quantitative analysis indicated that the binding specificity of $\mathrm{MB}_{\mathrm{MMP}-1}$ was significantly about 20 times higher than that of control $\mathrm{MB}_{\mathrm{IgG}}(298.42 \pm 16.57$ versus $12.38 \pm 3.26,{ }^{* *} P<0.01$ ) (Figure 4D).

\section{USMI In Vivo}

$\mathrm{MB}_{\mathrm{MMP}-1}$ and $\mathrm{MB}_{\mathrm{IgG}}$ were further evaluated in tumor-bearing mice at Day 7, Day 12, and Day 17 by USMI. The injections of $\mathrm{MB}_{\mathrm{MMP}-1}$ or $\mathrm{MB}_{\mathrm{IgG}}$ were separated by at least $30 \mathrm{~min}$. From Figure $\mathbf{5 A}$, we could clearly observe that the $\mathrm{MB}_{\mathrm{MMP}-1}$ of color red had more retention rate in tumors and significantly enhanced the US imaging signal intensity, while the control $\mathrm{MB}_{\mathrm{IgG}}$ showed a significantly lower enhancement than did $\mathrm{MB}_{\mathrm{MMP}-1}$. In addition, with the growth of LSCC, the dTE signal intensity was much higher in $\mathrm{MB}_{\mathrm{MMP}-1}$. From Figure 5B, we plotted the US dTE signal intensity from the LSCC versus growth time of the Day 7, Day 12, and Day 17. There were 20-, 10- and 8-folds higher $\mathrm{dTE}$ signal intensity from $\mathrm{MB}_{\mathrm{MMP}-1}$ than control $\mathrm{MB}_{\mathrm{IgG}}$. Then we quantify the dTE signal intensity with Vevo2100 inner software CQ. Quantification revealed $41.21 \pm 15.00$ a.u. for $\mathrm{MB}_{\mathrm{MMP}-1}$ versus $2.25 \pm 0.6$ a.u. for $\mathrm{MB}_{\mathrm{IgG}}$ at Day $7\left({ }^{*} P<0.05\right), 124.64 \pm 5.19$ a.u. $\mathrm{MB}_{\mathrm{MMP}-1}$ versus $11.13 \pm 1.13$ a.u. $\mathrm{MB}_{\mathrm{IgG}}$ at Day $12\left({ }^{\star} P<0.05\right)$, and $332.01 \pm 64.88$ a.u. $\mathrm{MB}_{\mathrm{MMP}-1}$ versus $42.99 \pm 11.9$ a.u. $\mathrm{MB}_{\mathrm{IgG}}$ at $\mathrm{Day}$ $17\left({ }^{* *} P<0.01\right)$.

\section{Tumor Immunofluorescence Staining}

To confirm the results of USMI using the $\mathrm{MB}_{\mathrm{MMP}-1}$, the tumor slices at Day 7, Day 12, and Day 17 were executed and subsequently analyzed for MMP-1 expression by immunofluorescence. Immunofluorescence results are shown in Figure 6; we could clearly see that the MMP-1 (red) was highly expressed in LSCC, confirming the presence of mouse MMP-1 on plasma membrane within LSCC in our study. In addition, we did the correlation analysis between the dTE signal intensity of USMI and mean optical density (/pixel) of MMP-1 expression in Figure 7; it was obvious that the dTE signal intensity of USMI was linearly enhanced as the mean optical density under the growth of the tumor. On the whole, the results of immunofluorescent evaluation at Day 7, Day 12, and Day 17 are relevant to the dTE signal intensity acquired with the $\mathrm{MB}_{\mathrm{MMP}-1}$ by USMI. 


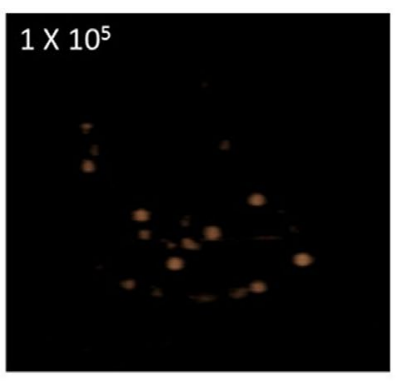

$\mathbf{A}$

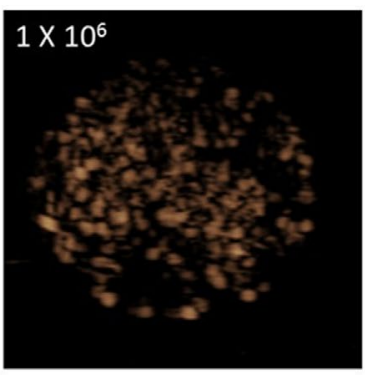

B

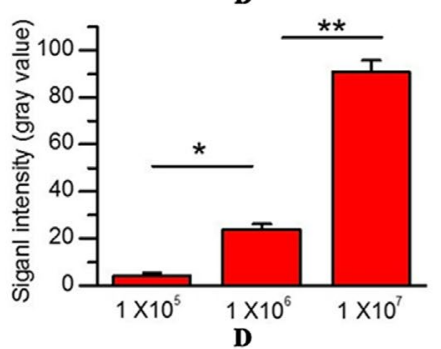

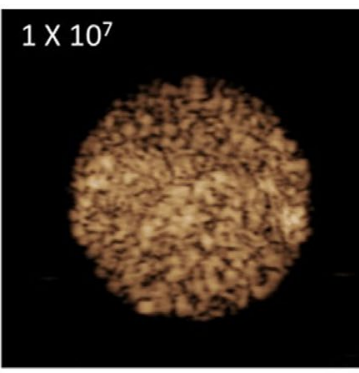

C

FIGURE 2 | In vitro US imaging performance. (A) $1 \times 105 \mathrm{MBs} / \mathrm{mL}$. (B) $1 \times 106 \mathrm{MBs} / \mathrm{mL}$. (C) $1 \times 107 \mathrm{MBs} / \mathrm{mL}$. (D) Quantitative analysis of US signal intensity of MBMMP-1 with three different concentrations.

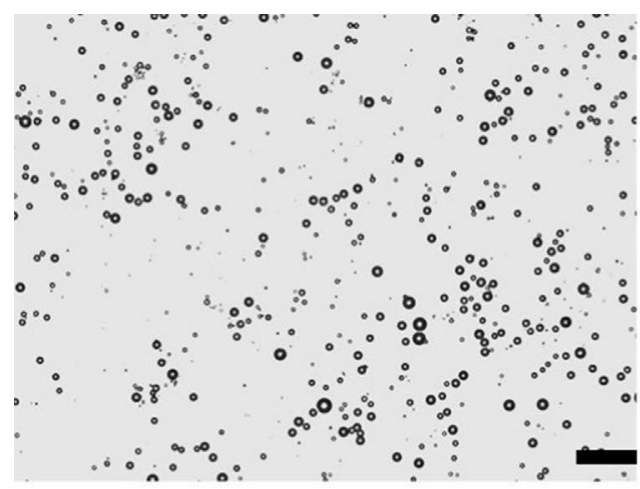

A

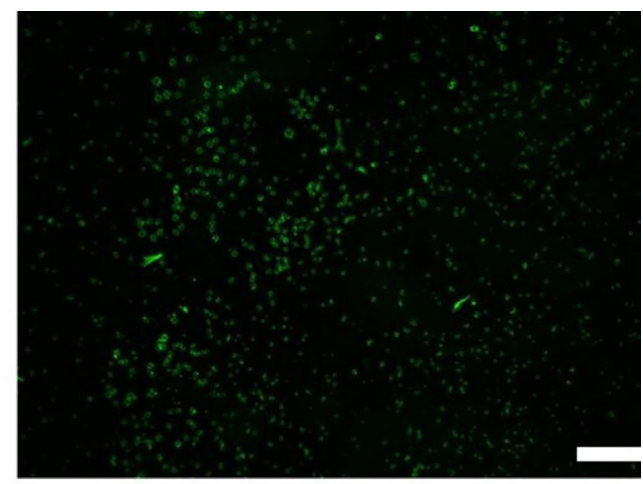

B

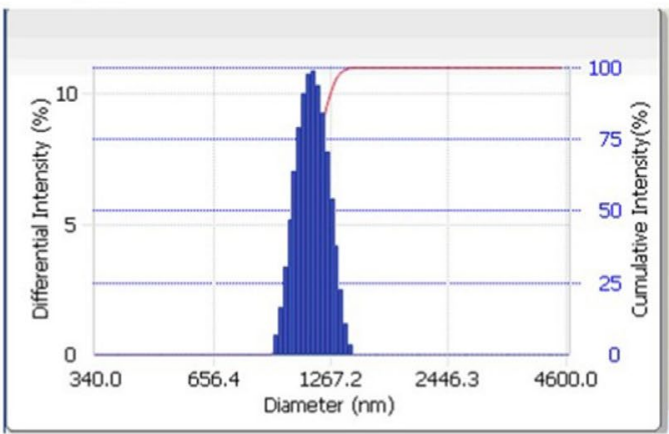

Distribution Results Peak Value (nm) $1.1137 .1 \pm 99.8$

Peak Avg. (nm)

$1137.1 \pm 99.8$

Avg. Residual

a $4306 . \mathrm{nn} 2$

\section{C}

FIGURE 3 | Characterization of $\mathrm{MB}_{\mathrm{MMP}-1 .}$. (A) Bright-filed photograph of $\mathrm{MB}_{\mathrm{MMP}-1 .}$ (B) Fluorescent micrograph of FITC-labeled MB $\mathrm{BMP}_{\mathrm{M}-1}$. (C) Average size and distribution of the $\mathrm{MB}_{\mathrm{MMP}-1}$ (scale bar $=10 \mu \mathrm{m}$ ). 

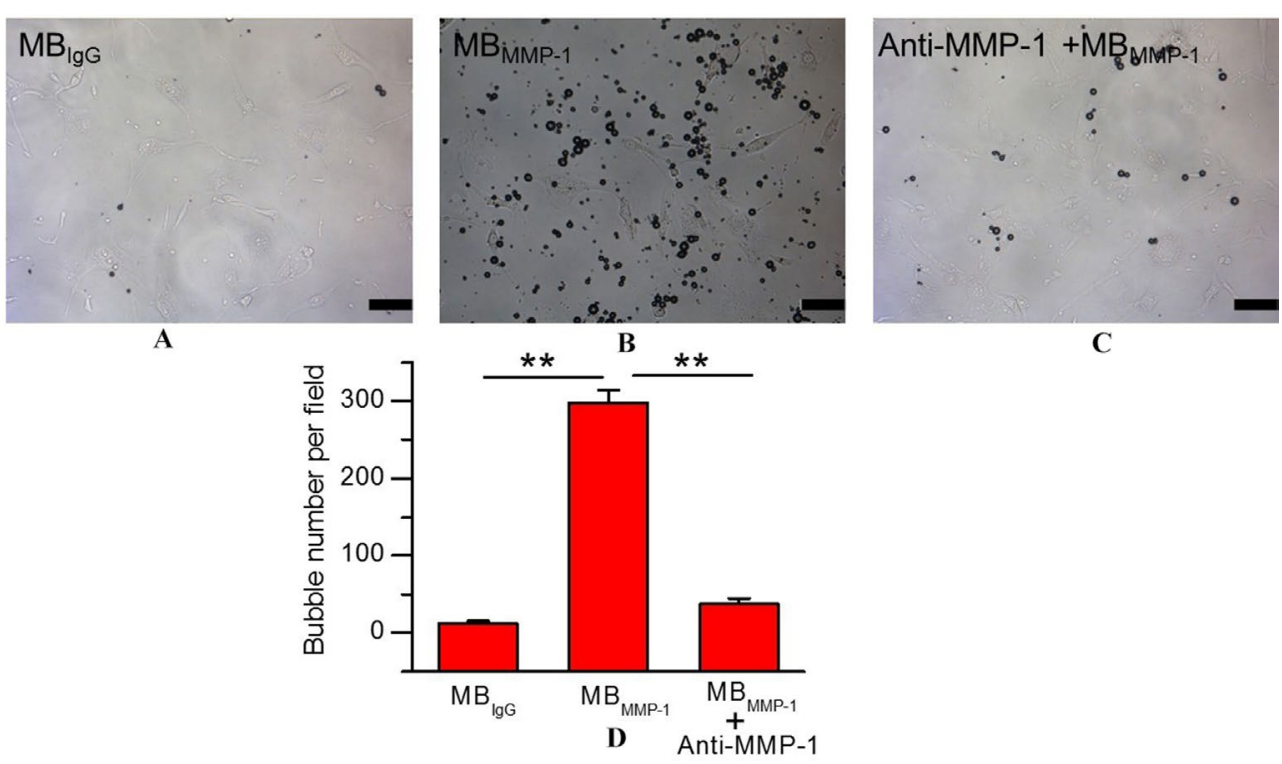

C

FIGURE 4 | In vitro binding specificity experiment of $\mathrm{MB}_{\mathrm{MMP}-1}$ to human epidermoid laryngeal cancer cells (HEp-2). (A) White light micrograph after incubating control group $\mathrm{MB}_{\mathrm{lgG}}$ with HEp-2 cells. (B) White light micrograph after incubating $\mathrm{MB}_{\mathrm{MMP}-1}$ with HEp-2 cells. (C) White light micrograph after incubating pre-blocked with free anti- matrix metalloproteinase-1 (MMP-1) antibody. (D) Quantitative analysis of the number of $\mathrm{MB}_{\mathrm{MMP}-1}$ and control group MB $\mathrm{Bg}_{\mathrm{gG}}$ that adhered onto HEp-2 from five random view fields $\left({ }^{* \star} P<0.01, n=5\right)$. Scale bar $=10 \mu \mathrm{m}$.
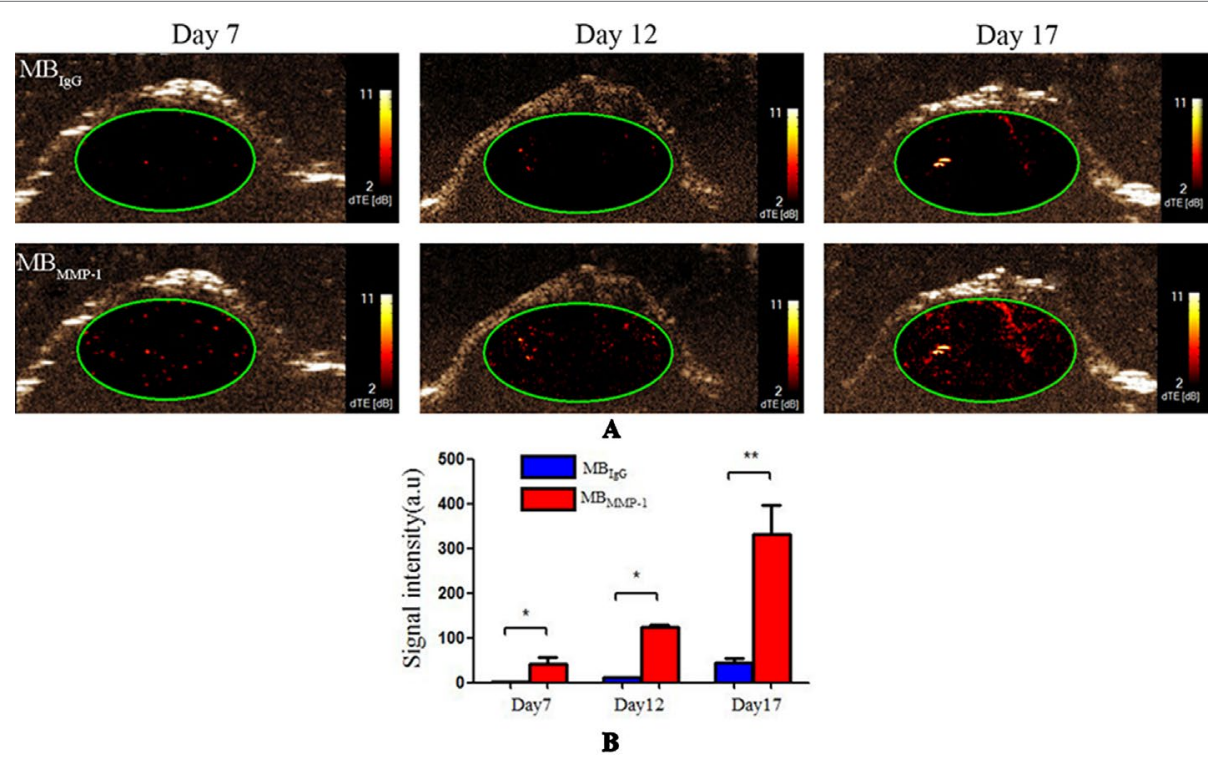

FIGURE 5 | Ultrasound molecular imaging (USMI) in vivo. (A) Color-coded differential targeted enhancement (dTE) signal sonograms separately at Day 7, Day 12, and Day 17. (B) Quantitative analysis of dTE for the $\mathrm{MB}_{\mathrm{MMP}-1}$ and control group $\mathrm{MB}_{\text {lgG }}\left({ }^{\star} P<0.05,{ }^{\star \star} P<0.01\right)$.

\section{DISCUSSION AND CONCLUSION}

Previous research had developed and tested arginine-glycineaspartate (RGD)-MBs using av $\beta 3$ integrin for a biomarker of the neovasculature in HEp-2 mouse tumor model (Hu et al., 2016). It had been proven that $\alpha v \beta 3$ integrin-targeted RGD-MBs could effectively assess the expression of neovasculature and enhance the contrast imaging signal. However, the specificity of $\alpha v \beta 3$ targeted MBs for LSCC detection was still not high enough because of its universality of expression in various types of malignant tumors (Sarmishtha et al., 2003; Brown et al., 2004; Hall and Jeffrey, 2004). Despite the growing number of published studies on USMI, a main obstacle for a clinical early diagnosis still exists. 


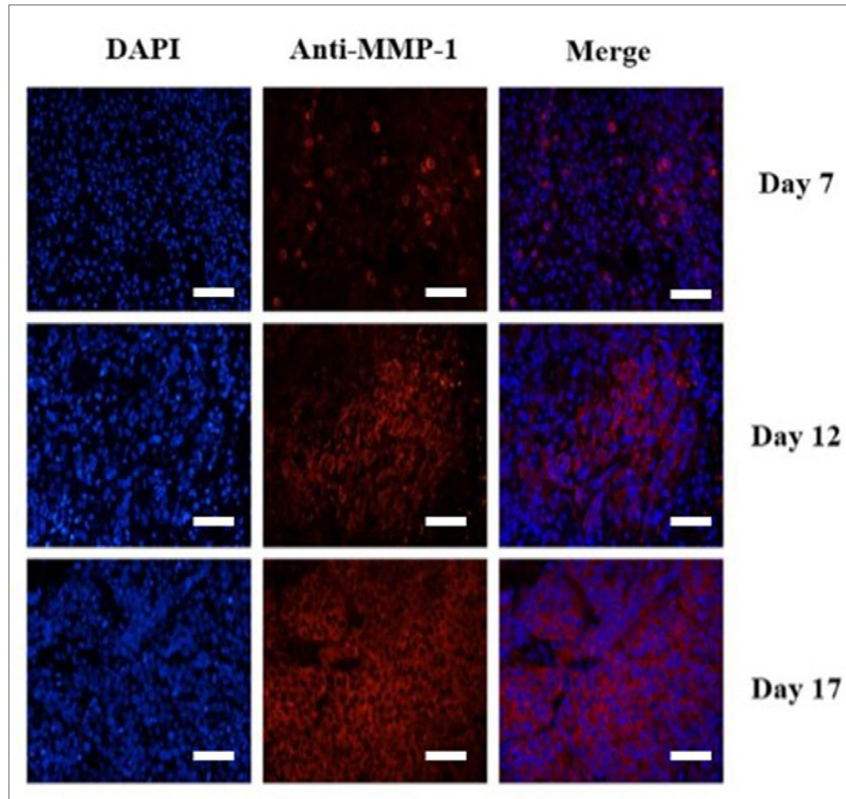

FIGURE 6 | Immunofluorescence staining of HEp-2 tumors for MMP-1 receptor. Immunofluorescence images of cell nucleus blue (first column), mouse MMP-1 red (second column), and merged (third column) MMP-1 and cell nucleus-stained image (the overlap of blue and red fluorescence confirms the co-localization of MMP-1) proved the expression of MMP-1 on plasma membrane in HEp-2 cells. MMP-1 was visualized with Cy3 dye (red). Cell nuclei were stained with DAPI (St. Louis, MO, USA) (blue). Scale bar $=200 \mu \mathrm{m}$.

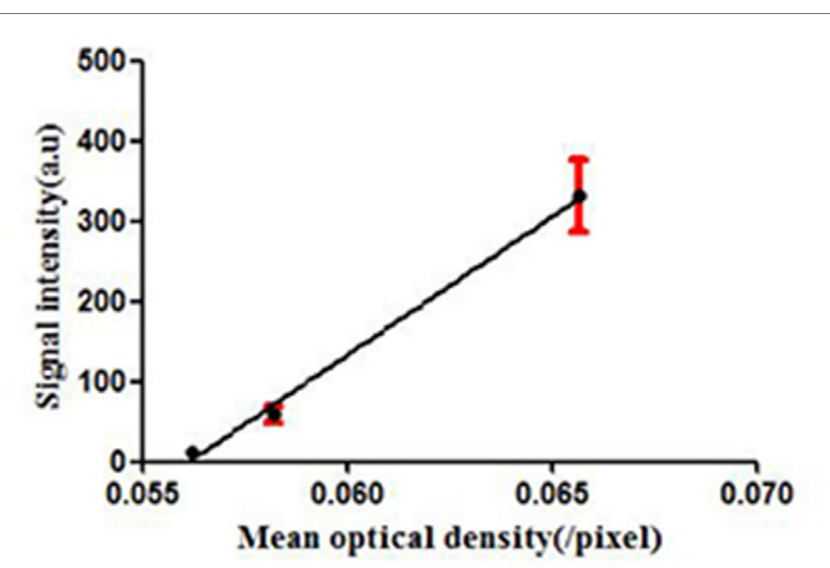

FIGURE 7 | dTE signal intensity of USMI and mean optical intensity (/pixel) images of tumor immunofluorescence staining separately at Day 7, Day 12, and Day 17.

In 1999, Maniotis and colleagues found the existence of VM in human melanoma cells (Maniotis et al., 1999). Subsequently, more and more studies had confirmed that VM is the new generation of tumor microcirculation without the participation of endothelial cells (Maniotis et al., 1999; Wang et al., 2010). And in tumor environment, it also was of importance to LSCC aggression and facilitates distant metastasis (Maniotis et al., 1999; Quail and Joyce, 2013). On the basis of these events, we assume that the lipid-shelled MBs loaded with mouse antiMMP-1 monoclonal antibodies could tightly gather around VM to evaluate the expression of MMP-1 in LSCC.
In our current study, a novel $\mathrm{MB}_{\mathrm{MMP}-1}$ USMI agent with $\mathrm{C}_{3} \mathrm{~F}_{8}$ filled lipid-shelled MBs were prepared to dynamically evaluate the expression of MMP-1 in LSCC separately at Day 7, Day 12, and Day 17. First, we found that the $\mathrm{MB}_{\mathrm{MMP}-1}$ had the ideal particle size range and that $1 \times 10^{8} \mathrm{MBs} / \mathrm{mL}$ concentration enhanced the US imaging signal in vitro. Then, we further confirmed the specific binding ability of $\mathrm{MB}_{\mathrm{MMP}-1}$ to $\mathrm{HEp}-2$. It revealed that $\mathrm{MB}_{\mathrm{MMP}-1}$ not only exhibited significantly greater adhesion to HEp-2 than did control $\mathrm{MB}_{\mathrm{IgG}}$ but also significantly provided a prospecting result for the next animal experiment. In vivo, we clearly found that the signal intensity of dTE of $\mathrm{MB}_{\mathrm{MMP}-1}$ group had significantly higher retention than did control $\mathrm{MB}_{\mathrm{IgG}}$ between each group. In addition, it is also noticeable that the signal intensity of dTE showed an upward trend with the time increasing of tumor. This result could be because a much more universal VM was newborn in the later period of LSCC than in the early period (Wang et al., 2010). A further study also confirmed that more and more newborn VM were increasing the participates in cancer progression and metastasis to supply tumor with sufficient nutrition (Hong and Hui, 2018). As expected, these in vivo dynamical evaluation results are further confirmed by immunofluorescence. From Figure 6, the red-stained MMP-1 receptors were much more expressed with the growth of tumors. This immunofluorescence analysis was well correlated with the results from signal intensity of dTE by USMI in vivo (Figure 7).

The following limitations of the study need to be solved. First, the size of MBs we chose is of microsize level, which can only indirectly draw support from no endothelial cells' VM by USMI. Second, although there was a strong significance in evaluating the expression of MMP-1 in LSCC, contrast agents adhering to more than one molecular biomarker may be advantageous over singletargeted contrast agents (Du et al., 2018) by increasing the number of MBs attached at sites of tumor neovascular and tumor tissue.

In conclusion, a novel $\mathrm{MB}_{\mathrm{MMP}-1}$ US contrast agent will lay the foundation for the application of target biomarker of LSCC for USMI and will be a promising method to improve the early diagnosis.

\section{ETHICS STATEMENT}

All animal studies were approved by the Institute's Animal Care and Use Committee of Shenzhen Second People's Hospital, Guangzhou University School of Medicine, China.

\section{AUTHOR CONTRIBUTIONS}

$\mathrm{QH}$ and ZL proposed the project. YZ and ZS conducted the study, $\mathrm{XJ}, \mathrm{HZ}$, and XW analyzed the data. YZ wrote the manuscript. QH revised the manuscript. All authors reached an agreement with the final version of the manuscript.

\section{FUNDING}

Thepresentstudyreceived financial supportfrom The National Natural Science Foundation of China (81660292) and Shenzhen Science and Technology Project (project numbers JCYJ20170817171836611 and JCYJ20170306092258717). The National Natural Science Foundation of China (81260223 and 81301300) and GuangXi medical high-level backbone personnel training " 139 " project also supported this study. 


\section{REFERENCES}

Abou-Elkacem, L., Wilson, K. E., Johnson, S. M., Chowdhury, S. M., Bachawal, S., Hackel, B. J., et al. (2016). Ultrasound molecular imaging of the breast cancer neovasculature using engineered fibronectin scaffold ligands: a novel class of targeted contrast ultrasound agent. Theranostics 6 (11), 1740-1752. doi: 10.7150/thno.15169

Balkwill, F. R., Capasso, M., and Hagemann, T. (2012). The tumor microenvironment at a glance. J. Cell Sci. 125 (23), 5591-5596. doi: 10.1242/jcs.116392

Brown, C. K., Khodarev, N. N., Yu, J., Moo-Young, T., Labay, E., Darga, T. E., et al. (2004). Libationary cells block radiation-induced programmed cell death of endothelial cells. FEBS Lett. 565 (1), 167-170. doi: 10.1016/j.febslet.2004.03.099

Castelijns, J. A., and Mw, V. D. B. (1993). Magnetic resonance imaging evaluation of extracranial head and neck tumors. Magn. Reson. Q. 9 (2), 113-128. doi: 10.1002/mrm.1910290620

Du, J., Li, X. Y., Hu, H., Xu, L., Yang, S. P., and Li, F. H. (2018). Preparation and imaging investigation of dual-targeted $\mathrm{C}_{3} \mathrm{~F}_{8}$-filled PLGA nanobubbles as a novel ultrasound contrast agent for breast cancer. Sci. Rep. 8 (1), 3887. doi: 10.1038/s41598-018-21502-X

Fabian, K., Stanley, F., Patrick, K., Wiltrud, L., and Twan, L. (2012). Ultrasound microbubbles for molecular diagnosis, therapy, and theranostics. J. Nucl. Med. 53 (3), 345-348. doi: 10.2967/jnumed.111.099754

Feng, Y., Zhi-Yi, C., and Yan, L. (2013). Advancement of targeted ultrasound contrast agents and their applications in molecular imaging and targeted therapy. Curr. Pharm. Des. 19 (8), 1516-1527. doi: 10.2174/138161213804805469

Gino, M., Filippo, M., Stella, B., Emiliano, D. A., Luciano, G., Vincenza, G., et al. (2010). Neoangiogenesis in laryngeal carcinoma: angiogenin and CD105 expression is related to carcinoma recurrence rate and disease-free survival. Histopathology 57 (4), 535-543. doi: 10.1111/j.1365-2559.2010.03664.x

Gupta, B., Johnson, N. W., and Kumar, N. (2016). Global epidemiology of head and neck cancers: a continuing challenge. Oncology 91 (1), 13-23. doi: $10.1159 / 000446117$

Hall, H., and Jeffrey, A. H. (2004). Matrix-bound sixth Ig-like domain of cell adhesion molecule $\mathrm{L} 1$ acts as an angiogenic factor by ligating av 333 -integrin and activating VEGF-R2. Microvasc. Res. 68 (3), 169-178. doi: 10.1016/j.mvr.2004.07.001

Hong, G., and Hui, L. (2018). Overview of advances in vasculogenic mimicry-a potential target for tumor therapy. Cancer Manag. Res. 10, 2429-2437. doi: 10.2147/CMAR.S164675

Hu, Q., Wang, X. Y., Kang, L. K., Wei, H. M., Xu, C. M., Wang, T., et al. (2016). RGD-targeted ultrasound contrast agent for longitudinal assessment of Hep-2 tumor angiogenesis in vivo. PLoS One 11 (2), e0149075. doi: 10.1371/journal. pone. 0149075

Kiessling, F., Huppert, J., and Palmowski, M. (2009). Functional and molecular ultrasound imaging: concepts and contrast agents. Curr. Med. Chem. 16 (5), 627-642. doi: 10.2174/092986709787458470

Kumar, C. C. (2003). Integrin alpha v beta 3 as a therapeutic target for blocking tumor-induced angiogenesis. Curr. Drug Targets 4 (2), 123-131. doi: $10.2174 / 1389450033346830$

Maniotis, A. J., Folberg, R., Hess, A., Seftor, E. A., Gardner, L. M., Pe'Er, J., et al. (1999). Vascular channel formation by human melanoma cells in vivo and in vitro: vasculogenic mimicry. Am. J. Pathol. 155 (3), 739-752. doi: 10.1016/ S0002-9440(10)65173-5

Norling, R., Buron, B. M., Therkildsen, M. H., Henriksen, B. M., Von, B. C., and Nielsen, M. B. (2014). Staging of cervical lymph nodes in oral squamous cell carcinoma: adding ultrasound in clinically lymph node negative patients may improve diagnostic work-up. PLoS One 9 (3), e90360. doi: 10.1371/journal. pone.0090360

Paolo, B., Locati, L. D., and Lisa, L. (2006). Biological agents in head and neck cancer. Ann. Oncol. 7 (11), 1643-1650. doi: 10.1586/14737140.7.11.1643

Pysz, M. A., Gambhir, S. S., and Willmann, J. K. (2010). Molecular imaging: current status and emerging strategies. Clin. Radiol. 65 (7), 500-516. doi: 10.1016/j.crad.2010.03.011

Quail, D. F., and Joyce, J. A. (2013). Microenvironmental regulation of tumor progression and metastasis. Nat. Med. 19 (11), 1423-1437. doi: 10.1038/nm.3394

Raffetto, J. D., and Khalil, R. A. (2008). Matrix metalloproteinases and their inhibitors in vascular remodeling and vascular disease. Biochem. Pharmacol. 75 (2), 346-359. doi: 10.1016/j.bcp.2007.07.004

Righi, P. D., Kopecky, K. K., Caldemeyer, K. S., Ball, V. A., Weisberger, E. C., and Radpour,S. (2015). Comparison of ultrasound-fine needle aspiration and computed tomography in patients undergoing elective neck dissection. Head Neck 19 (7), 604610. doi: 10.1002/(SICI)1097-0347(199710)19:7<604::AID-HED7>3.3.CO;2-K

Sarmishtha, D., Juhua, C., Narizhneva, N. V., Warren, H., Jennifer, B., E Helene, S., et al. (2003). Molecular pathway for cancer metastasis to bone. J. Biol. Chem. 278 (40), 39044-39050. doi: 10.1074/jbc.M304494200

Stieger, S. M., Dayton, P. A., Borden, M. A., Caskey, C. F., Griffey, S. M., Wisner, E. R., et al. (2010). Imaging of angiogenesis using Cadence contrast pulse sequencing and targeted contrast agents. Contrast Media Mol. Imaging 3 (1), 9-18. doi: 10.1002/cmmi.224

Tsukifuji, R., Tagawa, K., Hatamochi, A., and Shinkai, H. (1999). Expression of matrix metalloproteinase-1, -2 and -3 in squamous cell carcinoma and actinic keratosis. Br. J. Cancer 80 (7), 1087-1091. doi: 10.1038/sj.bjc.6690468

Upile, T., Jerjes, W., Radhi, H., Al-Khawalde, M., Kafas, P., Nouraei, S., et al. (2011). Vascular mimicry in cultured head and neck tumour cell lines. Head Neck Oncol. 3 (1), 55. doi: 10.1186/1758-3284-3-55

Wang, W., Lin, P., Han, C., Cai, W., Zhao, X., and Sun, B. (2010). Vasculogenic mimicry contributes to lymph node metastasis of laryngeal squamous cell carcinoma. J. Exp. Clin. Cancer Res. 29 (1), 60. doi: 10.1186/1756-9966-29-60

Wood, A. K. W., and Sehgal, C. M. (2015). A review of low-intensity ultrasound for cancer therapy. Ultrasound Med. Biol. 41 (4), 905-928. doi: 10.1016/j. ultrasmedbio.2014.11.019

Yamazaki, Y., Saitoh, M., Notani, K. I., Tei, K., Totsuka, Y., Takinami, S. I., et al. (2008). Assessment of cervical lymph node metastases using FDG-PET in patients with head and neck cancer. Ann. Nucl. Med. 22 (3), 177-184. doi: 10.1007/s12149-007-0097-9

Yan, F., Li, X., Jin, Q., Jiang, C., Zhang, Z., Ling, T., et al. (2011). Therapeutic ultrasonic microbubbles carrying paclitaxel and LyP-1 peptide: preparation, characterization and application to ultrasound-assisted chemotherapy in breast cancer cells. Ultrasound Med. Biol. 37 (5), 768-779. doi: 10.1016/j. ultrasmedbio.2011.02.006

Conflict of Interest Statement: The authors declare that the research was conducted in the absence of any commercial or financial relationships that could be construed as a potential conflict of interest.

Copyright (C) 2019 Zhou, Song, Hu, Ji, Zheng, Wang and Li. This is an open-access article distributed under the terms of the Creative Commons Attribution License (CC $B Y)$. The use, distribution or reproduction in other forums is permitted, provided the original author(s) and the copyright owner(s) are credited and that the original publication in this journal is cited, in accordance with accepted academic practice. No use, distribution or reproduction is permitted which does not comply with these terms. 